\title{
Editorial: Index Modulation for 6G Communications
}

\author{
Shuping Dang ${ }^{1 *}$, Marco Di Renzo ${ }^{2 *}$, Miaowen Wen ${ }^{3 *}$, Marwa Chafii ${ }^{4 *}$, Youngwook Ko ${ }^{5 *}$, \\ Bartolomeu F. Uchôa-Filho ${ }^{6 *}$ and Abdelhamid Younis ${ }^{7 *}$ \\ ${ }^{1}$ Department of Electrical and Electronic Engineering, University of Bristol, Bristol, United Kingdom, ${ }^{2}$ Université Paris-Saclay, \\ CNRS, CentraleSupélec, Laboratoire des Signaux et Systèmes, Gif-sur-Yvette, France, ${ }^{3}$ The School of Electronic and Information \\ Engineering, South China University of Technology, Guangzhou, China, ${ }^{4}$ The Division of Engineering, New York University (NYU) \\ Abu Dhabi, Abu Dhabi, United Arab Emirates, ${ }^{5}$ The Department of Electronic Engineering, University of York, York, \\ United Kingdom, ${ }^{6}$ The Department of Electrical Engineering, Federal University of Santa Catarina, Florianópolis, Brazil, ${ }^{7}$ Electrical \\ and Electronics Engineering Department, Faculty of Engineering, University of Benghazi, Benghazi, Libya
}

Keywords: index modulation, 6G, next generation networks, spectral efficiency (SE), energy effciency

\section{Editorial on the Research Topic}

\section{Index Modulation for 6G Communications}

Edited and reviewed by: loannis Krikidis,

University of Cyprus, Cyprus

*Correspondence: Shuping Dang

shuping.dang@bristol.ac.uk Marco Di Renzo

marco.direnzo@centralesupelec.fr Miaowen Wen

eemwwen@scut.edu.cn Marwa Chafi

marwa.chafii@nyu.edu Youngwook Ko

youngwook.ko@york.ac.uk Bartolomeu F. Uchôa-Filho uchoa@eel.ufsc.br Abdelhamid Younis a.alhassi@uob.edu.ly

Specialty section:

This article was submitted to Communications Theory,

a section of the journal Frontiers in Communications and Networks

Received: 13 October 2021 Accepted: 15 November 2021 Published: 03 December 2021

Citation:

Dang S, Di Renzo M, Wen M, Chafii M, Ko Y, Uchôa-Filho BF and Younis A (2021) Editorial: Index Modulation for 6G Communications.

Front. Comms. Net 2:794311. doi: 10.3389/frcmn.2021.794311
The past decades have witnessed surges in data throughput and the number of connected nodes throughout the fourth generation (4G) and fifth generation (5G) upgrades. Recent studies also forebode these increases in the future sixth generation $(6 \mathrm{G})$ networks, especially when the Industrial Internet of Things (IIoT) and space-air-ground network integration can be widely implemented in the 2030s. These tremendous increases will undoubtedly result in increasingly stringent requirements of spectral efficiency and energy efficiency of communication systems.

In recent years, index modulation (IM) has attracted researchers' attention as a means of meeting these two requirements. Different from conventional amplitude-phase modulation schemes, index modulation employs one or more index domain(s) in addition to the classic amplitude-phase constellations. Therefore, index modulation offers a means to form a higher-dimensional modulation scheme, which considerably enhances the spectral efficiency under proper system configurations. By index modulation, only a subset of transmit antennas/orthogonal subcarriers/time slots will be activated to form a unique activation pattern. Consequently, the index of activation pattern can be used to modulate extra bit streams, in addition to the bit streams modulated by constellation symbols. As a newborn paradigm in infancy, there are still a large number of open research questions awaiting solutions, and further research activities are of high importance to ultimately promote index modulation to the practical stage. More state-of-the-art communication technologies and the latest communication paradigms, e.g., terahertz bands, meta-surfaces, and cooperative relays, can be jointly applied with index modulation, which broadens its applicability.

In light of the aforementioned advantages, promising applications, and the good applicability brought by index modulation, the Guest Editors proposed this Research Topic to attract original and high-quality publications addressing the theoretical and practical issues related to index modulation for $6 \mathrm{G}$ communications. The Guest Editors would also like to take this Research Topic as an opportunity to stimulate further research activities related to index modulation in the $6 \mathrm{G}$ era. In total, four papers have been accepted for this Research Topic from scholars coming from a number of countries. The topics of these contributing papers cover a wide range of index modulation, including applying the index modulation concept in the context of cooperative communications, meta-surface based terahertz communications, massive random access networks, joint communication and radar-sensing systems.

The first accepted paper titled 'Toward Spectral and Energy Efficient 5G Networks Using Relayed OFDM With Index Modulation' by the researchers with King Abdullah University of Science and Technology (KAUST) is dedicated to index modulation in the frequency domain. In particular, this 
paper discusses the feasibility as well as the pros and cons of introducing cooperative relaying technology to help systems employing orthogonal frequency-division multiplexing with index modulation (OFDM-IM) harvest a diversity gain and raise energy efficiency. The recent achievements of such a joint paradigm have been summarized and appraised in this work. Besides, a full picture and some insightful viewpoints regarding the practical implementation of relay assisted OFDM-IM are also provided.

The second accepted paper titled 'Index Modulation-Aided Mixed Massive Random Access', by Liang et al. with Xidian University, analyzes index modulation in a broader context and considers massive random access scenarios, where users can be classified into two categories. A mixed massive random access scheme is proposed and studied in this work, which can support common information and user-specific information transmissions. Specifically, the common information is transmitted by index modulation aided unsourced random access (URA), while the user-specific information is transmitted by index modulation aided sourced random access (SRA). Employing both index modulation aided URA and SAR, the system error performance and complexity are illustrated and compared to conventional transmission schemes through numerical simulations.

The paper titled 'A Differential Modulation Scheme for Metasurface-Based Terahertz Communications' by Guo et al. extends the application of index modulation to meta-surfacebased terahertz communications. In this work, a differential spatial $\mathrm{THz}$ modulation (DSTM) scheme is proposed and analyzed for meta-surface-assisted terahertz communication systems. The index domain of the DSTM scheme is constructed upon the meta-surface pattern activation orders instead of the metasurface patterns per se. In this way, the systems adopting the DSTM scheme are able to perform differential detection and do not rely on the channel state information and the meta-surface response to incident terahertz signals. Simulations have been conducted to demonstrate the performance superiority of the proposed DSTM scheme in this paper.

The finale of this Research Topic, titled 'OFDM-IM for Joint Communication and Radar-Sensing: A Promising Waveform for
Dual Functionality', is contributed by Şahin et al. This work utilizes OFDM-IM for joint communication and radar-sensing purposes and particularly evaluates the OFDM-IM waveform in these emerging application scenarios. Extensive computer simulations are involved in this work to reveal the performance advantages of OFDM-IM waveform in comparison with the classical OFDM waveform with a fixed pilot design.

Before concluding this editorial, the Guest Editors would like to thank all the authors who contributed their manuscripts to this Research Topic and reported their latest research results. In the meantime, the Guest Editors would also like to thank all reviewers for their supports for reviewing the submitted papers to this Research Topic and providing their valuable comments and suggestions. A special note of thanks goes to Prof. MohamedSlim Alouini, the Field Chief Editor of Frontiers in Communications and Networks, for his continuous support and coordination for the administrative affairs of this Research Topic.

\section{AUTHOR CONTRIBUTIONS}

All editors involved in the structuring and writing of the editorial.

Conflict of Interest: The authors declare that the research was conducted in the absence of any commercial or financial relationships that could be construed as a potential conflict of interest.

Publisher's Note: All claims expressed in this article are solely those of the authors and do not necessarily represent those of their affiliated organizations, or those of the publisher, the editors and the reviewers. Any product that may be evaluated in this article, or claim that may be made by its manufacturer, is not guaranteed or endorsed by the publisher.

Copyright (c) 2021 Dang, Di Renzo, Wen, Chafii, Ko, Uchôa-Filho and Younis. This is an open-access article distributed under the terms of the Creative Commons Attribution License (CC BY). The use, distribution or reproduction in other forums is permitted, provided the original author(s) and the copyright owner(s) are credited and that the original publication in this journal is cited, in accordance with accepted academic practice. No use, distribution or reproduction is permitted which does not comply with these terms. 